\title{
HISTOIRE DES ARTS VISUELS
}

Manuel Insolera et Lydia Salviucc Insolera, La Spiritualité en images aux PaysBas méridionaux dans les livres imprimés des $x v^{\ell}$ et $x v I^{\ell}$ siècles. Louvain, Peeters, 1996. $16 \times 22,217$ p., index (Miscellanea Neerlandica, XIII).

Marc Fumaroli, L'École du silence. Le sentiment des images au xvir siècle. Paris, Flammarion, 1994. $15 \times 24,510$ p., index.

Michel Beurdeley, Peintres jésuites en Chine au xvII $I^{e}$ siècle. Paris, Anthèse, 1996. $26 \times 28,215 \mathrm{p}$.

Leandro Karnal, Teatro da fé. Representação religiosa no Brasil e no Mexico do seculo xv. Sao Paulo, Hucitec, 1998. $14 \times 20,253$ p.

Andrea Pozzo. Éd. Alberta Battistr. Milan, Luni, 1996. $25 \times 34,476$ p.

La bibliographie récente dans le domaine, largement défini, des arts visuels, incluant l'architecture et le thêâtre, produit les apparences d'un grand éclatement. Pas de grande synthèse, ni pour ce qui concerne un art déterminé, ni pour ce qui concerne une période, voire même un lieu géographique précis. Une myriade d'esquisses monographiques, souvent sous forme d'articles, principalement en Italie et aux États-Unis, dont il serait difficile de dresser ici un bilan significatif. On pourrait, abruptement, résumer la situation ainsi : l' « art jésuite " n'existe plus (même sous la forme du débat, souvent rhétorique, qui occupa les historiens de l'art jusqu'aux années 1960 - dans l'héritage de Carlo Galassi-Paluzzi - sur l'existence réelle d'un tel art $^{1}$ ); l'art des jésuites n'existe pas encore, conçu non pas comme un répertoire et un lignage de formes et de figures, mais comme l'ensemble des manifestations visuelles dont la Compagnie de Jésus, fortement investie, à l'échelle mondiale, sur le terrain des représentations culturelles, a environné ses activités, proprement apostoliques ou pédagogiques, civiques, etc. L'art des jésuites

1. Le recueil dirigé par Rudolph WITTKOWER, Baroque Art. The Jesuit contribution, New York, 1972, relève encore de cette catégorie, bien qu'on y trouve des développements beau coup plus neufs, sur la place de la discussion sur la légitimité de l'ornementation des églises dans les « Sermons domestiques » du général des jésuites Gian Pietro Oliva, contemporain de la construction (et de la décoration) de l'église romaine San-Ignazio. Gauvin A. Balley fait bien apparaître la place de ce recueil dans l'historiographie de l'art jésuite, in The Jesuits. Cultures, sciences, and the arts, op. cit. supra, p. 433-435.

Revue de synthèse : $4^{c}$ S. $\mathrm{n}^{\text {os }}$ 2-3, avr.-sept. 1999, p. 462-468. 
n'existe pas encore ${ }^{2}$, mais il n'est pas certain non plus qu'il n'ait pas vocation à l'éclatement, tout au moins du point de vue de l'histoire des formes, voire, plus largement, des choix thématiques et des sources culturelles. On ferait volontiers le pronostic pour les années à venir, en fonction des indices dont nous disposons, d'une recomposition de l'historiographie autour de trois nouveaux objets principaux, appelés par le passage de l'art jésuite à l'art des jésuites : tout d'abord, quelle pensée dans la première Compagnie de Jésus et, plus profondément, quelle pensée avec l'image, quelle expérience de l'image dans la pensée des nombreux théoriciens jésuites qui se sont prononcés, théologiquement et liturgiquement, sur le problème de la vénération des saintes images? Ensuite, comment rendre compte, dans les systèmes décoratifs jésuites, de ce que Giovanni Careri a appelé, à propos des chapelles du Bernin (dont celle de l'église jésuite San-Andrea-del-Quirinal ${ }^{3}$ ) un « montage des arts " et peut-on, à un certain niveau, repérer les mobiles d'une faveur particulière accordée, dans la Compagnie de Jésus ou autour d'elle, à l'enchaînement combiné des formes architecturales, sculpturales, picturales? Enfin, la fonction ou, plus modestement (et prudemment), la place des images dans l'entreprise missionnaire ne serait-elle pas le lieu possible, dans les recherches futures, d'une articulation entre l'extrême diversité des formes attachées aux pratiques jésuites et quelque chose comme un fondement et une justification de cette diversité, et de la culture de cette diversité, l'adaptation missionnaire?

On achèvera cependant ce parcours par l'évocation d'un quatrième domaine de recherches, en essayant de montrer rapidement pourquoi ce dernier concentre les difficultés de la définition d'un art jésuite et pourquoi, en même temps - selon plusieurs travaux récents - il semble possible d'y découvrir les traces, dans l'image, d'une pratique spécifique de l'image: l'illustration des livres imprimés.

\section{L'expérience des images}

Rappelons d'abord, bien que la date de cette publication soit antérieure à la limite chronologique de ce compte rendu, l'importance contribution de Valeria de Laurentiis, "Imagini ed arte in Bellarmino ", au recueil Bellarmino e la Contro-Riforma (éd. Romeo de Maio et al., Sora, Centro di studi Sorani Vincenzo Patriarci, 1990), qui s'efforçait de contextualiser les interventions de Robert Bellarmin sur le sujet de l'image, dans l'immédiate postérité du concile de Trente, en réimmergeant un discours de ce type, essentiellement théologique et liturgique, au sein des problématiques esthétiques et artistiques de son temps. C'est à une même tentative que se livre Walter Melion, avec une extrême attention au détail du texte et aux occurrences effectives des images positives, telles qu'elles furent contemplées, bref à l'expérience des images vécues, dans les travaux qu'il a récemment consacrés (en

2. Saluons d'autant mieux l'heureuse initiative d'Alain Tapié, conservateur du musée des Beaux-Arts de Caen : l'exposition qu'il prépare pour 2001 sur Les Flux baroques et le rôle des jésuites dans la peinture au $x v r^{e}$ siècle a justement pour ambition de reconstituer le cadre pictural des églises, des collèges et des maisons de la Compagnie de Jésus dans l'ensemble de ses provinces d'Europe. Signalons aussi, dans une perspective également ouverte, le catalogue de la récente exposition de Munich, Rom in Bayern. Kunst und Spiritualität der ersten Jesuiten, éd. Reinhold Baumstark, Munich, Hirmer, 1997.

3. Giovanni Careri, Envols d'amour, Paris, Usher, 1991. 
particulier dans le recueil The Holy Face and the paradox of representation, éd. Herbert L. Kessler et Gherardt Wolf, Baltimore, MD, Johns Hopkins University, 1997) aux Adnotationes et Meditationes de Jérôme Nadal, en 1595, texte conçu en regard d'un projet d'illustration du récit évangélique (les Evangelicae Historiae Imagines, d'abord publiées d'une manière autonome, en 1593, avant d'être rattachées aux Adnotationes en 1596). Les recherches de Melion présentent en particulier l'avantage, par rapport à d'autres travaux attachés au même corpus ${ }^{4}$, de prendre le contre-pied des problèmes liés à un projet iconographique dont il est très difficile de savoir quelle part exacte y a prise son « auteur » final (mort depuis près de quinze ans - en 1580 - au moment de la publication des ouvrages mentionnés), en montrant comment, dans le commentaire effectivement rédigé par Nadal (l'un des plus proches collaborateurs d'Ignace de Loyola, d'où, évidemment, l'importance du dossier pour ce qui concerne l'enracinement d'une «politique de l'image » dans la toute première histoire de l'Ordre), la référence visuelle intervenait avec une grande précision et commandait directement l'orientation d'un discours par ailleurs dominé par une glose théologique du récit évangélique. Ce contrepied est essentiel, car il fait revenir, par la fenêtre du texte, en quelque sorte, un objet - l'image - dont il fallait aussi montrer, ce à quoi je me suis essayé dans l'ouvrage cité, qu'il n'était pas d'évidence conçu (comme la tradition l'a longtemps laissé penser) comme un complément naturel de la pratique des Exercices spirituels d'Ignace de Loyola. Il faut enfin, dans ce même chapitre, insister sur un moment important de la vie artistique post-tridentine, auquel l'édition récente, très bien documentée, de l'ouvrage majeur - et méconnu - de Francisco Pacheco, publié après la mort de son auteur, en 1644, El Arte de la pintura (éd. Bonaventura Bassegoda i Hugas, Madrid, Catedra, 1990) ouvre désormais un accès renouvelé ${ }^{5}$ : l'activité de l'académie de Séville, très proche de la Compagnie de Jésus, et simultanément engagée - à travers cette Compagnie - sur le terrain théorique de l'élaboration des normes de la bonne peinture catholique et sur le terrain pratique de la commande des cuvres : où l'on retrouve du reste - à une échelle qui reste aujourd'hui inexplorée - une double influence des Evangelicae Historiae Imagines de Nadal, d'une part en tant que modèle idéal des œuvres à prescrire, d'autre part en tant que patron effectif offert à l'imitation, pour ces mêmes ouvres. Dans cet intervalle, se trouve exposé l'enjeu d'une autonomie relative des «théoriciens » de la Contre-Réforme - où comment inventer ce qui est, produire comme possible ce qui est donné -, qui ouvre, nous semble-t-il, un champ de recherches considérables, aussi bien pour le repérage de la force des images à l'œuvre dans le discours que pour l'approfondissement de la distance d'une pensée jésuite de l'image par rapport à des problématiques strictement iconographiques. L'œuvre de Pacheco donne, de plus, les moyens du rééquilibrage, au bénéfice de l'Espagne, d'une bibliographie jusqu'ici largement centrée, autour de ces questions, sur l'Italie (avec les travaux liés au célèbre Discours sur les images sacrées et profanes de Gabriele

4. Je me permets de renvoyer ici à Pierre-Antoine FaBre, Ignace de Loyola. Le lieu de l'image, Paris, Vrin, 1992, qui rappelle les principales études sur le sujet.

5. Citons également la traduction française récente de l'ourvrage de Jonathan Brown, Images et idées dans la peinture du siècle d'or, Paris, Gérard Monfort, 1993, dont l'un des principaux chapitres est entièrement consacré à l'académie de Séville dans la première moitié du XVII siècle. 
Paleotti, en 1582) ou sur les Flandres (avec la réédition récente du Traité sur les saintes images de Johannes Molanus, 1570, Paris, Cerf, 1997). C'est entre ces pôles qu'il faut aujourd'hui insérer l'ensemble des positions prises par les auteurs liés à la Compagnie de Jésus sur le sujet des images, et sur les conditions de possibilité de leur production et de leur usage, face aux contestations de la Réforme, mais aussi face aux ambivalences du catholicisme moderne devant la représentation figurée des mystères de la foi.

\section{Le « montage des arts»}

La circulation entre les arts - au premier rang desquels l'architecture ${ }^{6}$, la sculpture et la peinture (la gravure appelant, nous semble-t-il, un développement spécifique) - dans la culture jésuite depuis la fin du XvI siècle, mais surtout au $x{ }^{e}{ }^{e}$, peut être saisie à trois niveaux d'analyse : tout d'abord, à celui, très général, de l'épreuve d'une traductibilité des formes les unes dans les autres et de la continuité, dans cette traduction, d'un même corpus symbolique - par exemple, la vie des saints fondateurs; puis, au niveau économique (qui porte avec lui, dans la première période, le principe d'une certaine retenue des investissements artistiques de l'Ordre) : il s'agit d'employer, voire de former, un personnel polyvalent, apte à toutes les virtuosités, depuis la décoration des voûtes d'église jusqu'à l'ornementation des confessionaux. Enfin, à un troisième niveau, théologique et spirituel, celui par lequel se trouve garanti, à travers la continuité du passage entre la peinture et l'architecture d'un côté, entre l'architecture et la peinture de l'autre, la légitimité de l'édifice comme sanctuaire de l'image sacrée et de l'image comme tapisserie du temple. Le deuxième niveau d'analyse, qui réclamerait une prosopographie systématique des premiers artistes de l'Ordre, qu'ils en soient membres ou non (mais beaucoup le furent), reste aujourd'hui un chantier ouvert, pour lequel le P. Pietro Pirri, auteur de nombreux ouvrages classiques sur les premiers architectes jésuites, aujourd'hui disparu, avait multiplié les «préparatifs » archivistiques ${ }^{7}$. Mais, pour l'ensemble du problème, la publication en 1996 d'un monumental ouvrage sur Andrea Pozzo - un artisan savant aussi bien qu'un artiste - a certainement été un événement décisif ${ }^{8}$ : non seulement parce que, en mobilisant une équipe de spécia-

6. Signalons au passage, de L. Patetta et S. Della Torre, L'Architettura della Compagnia di Gesú in Italia, xvI-xvI, Gênes, 1992, moins ambitieux, sans doute, que la somme sur Andrea Pozzo (voir infra, n. 8), mais qui propose un catalogue utile, très illustré, des édifices de la Compagnie à travers le monde.

7. Pour ce qui concerne l'architecture, la publication des sources est entrée dans une ère nouvelle, en Italie, avec le premier volume de Richard BöseL, Jesuiten Architektur in Italien, 1540-1773, Vienne, 1985.

8. L'ouvrage renvoie d'un coup loin dans le passé deux tendances longtemps dominantes dans la bibliographie de Pozzo (essentiellement centrée, d'ailleurs, sur les décorations romaines) : d'une part, Pozzo maître du trompe-l'œil, d'autre part, et complémentairement, Pozzo peintre spirituel « à programme », p. ex. dans l'étude de B. Prior, Parallel structures in Pozzo's triumph of san Ignatius and the Spiritual Exercises, dactylogr., Rome, 1977. Il encourage de nouvelles approches «globales» des sites jésuites, comme en témoigne l'étude récente d'Evonne LEvy (par ailleurs collaboratrice du volume sur Pozzo) sur le sanctuaire de Stanislas Koska, « jeune saint » jésuite des premières années au xva' siècle, proche de l'église San-Andrea-al-Quirinal, « Reproduction in the cultic era of art. Pierre Le Gros statue's of san Koska », Representations, printemps 1997, p. 88-113. 
listes extrêment diversifiée ${ }^{9}$, il parvient à traiter, pour la première fois, de toute l'activité de Pozzo (auquel, bien qu'il ne fût que le frère Pozzo, le général de la Compagnie - le P. Charles Noyelle - fit don, entre 1681 et 1686 , des premières esquisses préparatoires aux Evangelicae historiae imagines de Nadal), c'est-à-dire à la fois de ses différentes productions, picturales, sculturales et architecturales, pratiques et théoriques (puisque la célébrité de Pozzo est sans doute d'abord due à son traité de perspective, La Perspective propre des peintres et des architectes, selon le titre de l'édition française de 1700), et des multiples chantiers de cette production en Italie et en Europe (Pozzo meurt à Vienne en 1709); mais aussi parce que les études réunies montrent, sous chaque angle, comment la totalité des « vertus » de l'artiste fut mobilisée, par exemple dans la conception des architectures éphèmères, ou dans la décoration du vestibule d'accès aux camerette romaines d'Ignace de Loyola, magnifiquement photographiée dans le livre et qui met en jeu le rapport d'une « très ancienne Madone » peinte (en trompe-l'œil) et d'une architecture (feinte elle aussi) dans laquelle cette image sacrée s'inscrit ${ }^{10}$.

Indiquons pour finir que le développement actuel des recherches sur le théâtre des collèges jésuites, auquel la publication, en cours en Italie, des travaux de Bruna Filippi, d'une part sur le Collège romain (avec une approche très articulée de tous les aspects, pédagogiques, rhétoriques, esthétiques, politiques, rituels, du « théâtre » jésuite), d'autre part sur l'ensemble du répertoire dramatique des collèges italiens au $\mathrm{XVII}^{\mathrm{e}}$ siècle, à partir du dépouillement d'un grand nombre de sources inédites, apportera une importante contribution, s'inscrit fortement dans cette dynamique " protéiforme ${ }^{11} "$.

\section{L'image missionnaire}

On ne peut que mentionner très rapidement ici, tant le chantier est vaste, et les réalisations éparses, la place centrale que devrait recevoir, et que recevra sans doute dans les prochaines années à la faveur du renouveau général des études d'histoire missionnaire (auxquelles d'autres « synthèses " sont consacrées dans ce numéro), l'analyse du rôle des images, au titre des méthodes de l'évangélisation, et en tant

9. Viktor Gramatowski, p. ex., retrace la carrière de Pozzo à travers les jugements produits sur lui par ses supérieurs et conservés dans les « catalogues » de l'Ordre (ou, plus indirectement, dans les correspondances); Lydia Salviucci étudie, dans le texte et dans l'image, les éditions du Traité de perspective), Cristina Mossetti les tableaux réalisés pour la chapelle de la confrérie turinoise des banquiers et des marchands, etc. - toutes études qui, par la précision de leur documentation, la qualité de leur illustration et la finesse de leur approche constituent, à chaque fois, des instruments de référence.

10. Signalons d'ailleurs que la décoration de ce vestibule a été entièrement redécouverte dans le cadre d'une restauration récente, et que la " très ancienne Madone " avait été dissimulée par des réaménagements antérieurs des surfaces peintes.

11. Le recueil d'études, d'un intérêt inégal pour notre propos, qui porte le titre $l$ Gesuiti e $i$ primordi del teatro barroco in Europa, éd. M. Chiabo, F. Doglo, Rome, Centro studi sul teatro medioevale e rinascimentale, 1995, reste aujourd'hui un instrument utile pour le repérage des principales orientations de la recherche actuelle dans ce domaine, en Italie surtout. On retiendra, en particulier, les articles de Daniela Quarta sur le répertoire du Collège romain, de Nigel Griffith sur l'expurgation des auteurs latins et, du même auteur, en fin de volume, un bon guide bibliographique européen. 
que vecteur d'infiltration et d'échange matériel, culturel et symbolique. Le paysage reste ici très ouvert, entre les tentatives anciennes de Miguel Nicolau, dans le cadre de ses recherches sur Nadal ${ }^{12}$, pour mesurer la portée des " conversions " chinoises des gravures flamandes des Imagines, conversion formelle (et traduction) de l'image, conversion à l'image (à un certain modèle narratif), volonté de conversion par l'image et la mobilisation actuelle, du côté du concept de « métissage », dans les travaux de Serge Gruzinski par exemple, d'une vaste culture au sein de laquelle l'inscription spécifique des stratégies missionnaires reste à construire. On a choisi ici, au titre d'exemple de l'ampleur du champ, de faire apparaître, parmi les publications récentes, d'une part l'ouvrage consacré par Michel Beurdeley aux Peintres jésuites en Chine, qui donne à voir la manière dont l'activité des artistes jésuites est une sorte de miroir tendu à la réalité et aux représentations qui les environnent, et comment toute notion d'une «conversion par l'image » doit être réfléchie dans ce miroir, un réfléchissement dont le décryptage idéologique et apostolique demeure difficile en fonction même de son principe; d'autre part, dans la continuité de nos remarques précédentes sur le thêâtre, la passionnante étude d'un jeune chercheur brésilien, Leandro Karnal, Teatro da fe, qui, du Brésil au Mexique (circulation précieuse et rare, dans un domaine fortement exposé au risque d'une spécialisation géographique rapide), montre comment la représentation jésuite (mais pas seulement, autre vertu de l'ouvrage) des mystères religieux ouvre un espace dans lequel les voies de l'évangélisation prennent le détour risqué d'un merveilleux artifice.

\section{L'imprimé illustré}

Le domaine de la production des imprimés illustrés est certainement l'un de ceux dans lesquels la recherche actuelle est la plus vivante et la plus prometteuse. À l'appui (entre autres travaux) des Livres d'emblèmes et de devises. Une anthologie (1531-1735), de Jean-Marc Chatelain (Paris, Klincksieck, 1993) et dans l'attente des résultats de la vaste enquête entreprise par Ralph De Koninck, pour le Fonds national de la recherche en Belgique, sur l'ensemble du corpus gravé lié à la commande jésuite aux Pays-Bas aux XVI et $\mathrm{XVII}^{\mathrm{e}}$ siècles $^{13}$, on peut proposer l'hypothèse suivante, largement nourrie par l'importante publication de Manuel Insolera et Lydia Salviucci Insolera, La Spiritualité en images aux Pays-Bas méridionaux dans les livres imprimés des $x v f^{e}$ et $x v I^{\circ}$ siècles, qui s'efforce de conduire parallèlement l'enquête sur « Le livre spirituel en général » et sur «Le livre spirituel jésuite en particulier » (l'ouvrage propose de plus un remarquable catalogue, techniquement précis et culturellement ouvert, des principales « pièces à conviction » dans les deux domaines, en particulier, pour le second, sur Jérôme Nadal - et ses nombreux graveurs -, Bartolomeo Ricci - et le graveur Adrianus Collaert -, Antonius Sucquet

12. Miguel Nicolau, Jeronimo Nadal, sus obras y doctrinas espirituales, Madrid, Consejo superior de investigaciones cientificas, 1949; voir aussi, dans cette même période, Pasquale D'Eus, Le Origini del arte christiano chinese, Rome, Reale Accademia d'Italia, 1939 : ces dates disent avec éloquence le retard des recherches, en particulier sur le sujet de la gravure, qui relève encore, dans ses ouvrages, d'une approche " militante ".

13. Il faut y ajouter l'intéressant article de Kristeen NoreEN, "Ecclesia Militantis Triumphi. Jesuit iconography and the Counter-Reformation ", Sixteenth-Century Journal, vol. XXIX, 3, 1998. 
- et le graveur Boetium a Bolswert -, Hermannus Hugo, Giulelmus Hesius, etc.) : l'image gravée assume - y compris dans le rapport étroitement polémique qu'elle entretient avec l'iconographie protestante, dont la gravure fut, comme on le sait, un vecteur privilégié - ce que l'on pourrait appeler une « sortie du temple », en même temps qu'elle est le lieu essentiel d'un nouveau réglage, dans le contexte d'une diffusion progressivement massive de l'écrit, entre le texte - et, au premier chef, le texte sacré sous les nouvelles formes de sa transmission - et son illustration. D'où le fait qu'elle puisse simultanément jouer le jeu de l'émancipation, dont la feuille volante serait la figure extrême, mais dont les rapports complexes de l'image et du texte au sein des imprimés illustrés proposent des variantes multiples, et supporter la tentative d'un contrôle, c'est-à-dire inscrire dans ses dispositifs de lecture (le terme étant ici employé à dessein, étant donné le rôle, dans l'ensemble de ces recherches, de l'articulation du visible et du lisible, très concrètement de l'image et de ses légendes) la marque d'une orientation de la pratique de l'image qui n'est plus arrimée au lieu de l'église et doit inventer un autre lieu pour la cortemplation. D'où cet autre fait, complémentaire, que l'univers de ces gravures puisse tout à la fois aussi difficilement donner prise à des procédures d'identification iconographique, par exemple, et provoquer la mise en évidence d'autres formes de légitimation. La gravure devient alors probablement la voie par laquelle le renoncement à la définition d'un « art jésuite » n'implique pas le renoncement à la recherche, « hors-les-murs », d'une pratique jésuite de l'image dont certaines gravures porteraient la trace - et spécialement celles qui, dans les premières décennies de l'histoire de l'Ordre, s'accompagnent d'une forte résistance, au sein de la Compagnie elle-même, à la décoration de ses églises. L'ensemble de ces hypothèses est en débat. Ce débat impliquerait, par exemple, une discussion de l'histoire des frontispices des imprimés illustrés jésuites, telle que la présente, dans le chapitre fortement charpenté qu'il lui consacre dans son École du Silence, Marc Fumaroli, qui repère un processus au terme duquel « le frontispice devient la première illustration de l'ouvrage », alors que d'autres lectures sont possibles : celles-ci feraient au contraire des frontispices, depuis la fin du $x v{ }^{e}$ siècle, le lieu d'une confrontation entre le texte et l'image, le lieu d'un jeu par lequel le frontispice «virtualise » l'image en même temps que l'image - l'horizon du texte comme support visible - s'y donne comme le support du texte; dans ce jeu, le frontispice « ne devient la première illustration de l'ouvrage » que dans le moment même où l'image se découvre comme le lieu du texte. L'une et l'autre partie se trouvent liées entre elles dans un rapport nécessairement ouvert et réversible. C'est du point de vue de cette nécessité, selon moi, que les ambivalences du «non art jésuite " trouvent dans la gravure, et tout spécialement dans les imprimés illustrés, un espace de déploiement au sein duquel les images peuvent elles-mêmes parler. 